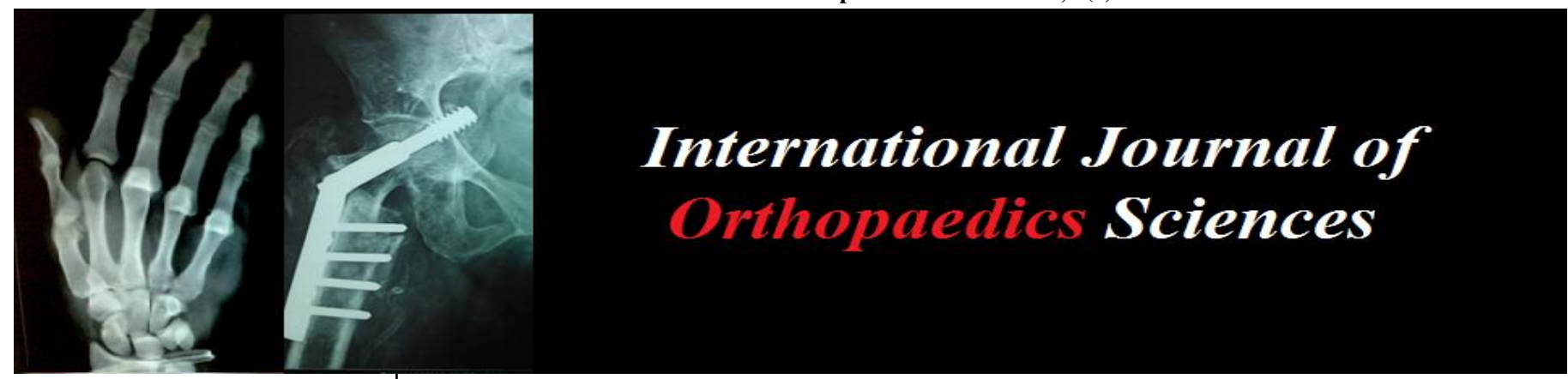

E-ISSN: 2395-1958

P-ISSN: 2706-6630

IJOS 2021; 7(2): 495-498

(C) 2021 IJOS

www.orthopaper.com

Received: 07-02-2021

Accepted: 13-03-2021

Dr. Mahendra Prakash Jain Department of Orthopaedics, RNT Medical College MB Hospital, Udaipur, Rajasthan, India

Dr. Deepak Agrawal Department of Orthopaedics, RNT Medical College MB Hospital, Udaipur, Rajasthan, India

Dr. Dheeraj Yadav

3rd Yr. Resident, Department of Orthopaedics, RNT Medical

College MB Hospital, Udaipur, Rajasthan, India
Corresponding Author: Dr. Mahendra Prakash Jain Department of Orthopaedics, RNT medical college MB hospital, Udaipur, Rajasthan, India

\section{Significance of platelet rich plasma (PRP) and Corticosteroid injection in management of Adhesive capsulitis of shoulder}

\section{Dr. Mahendra Prakash Jain, Dr. Deepak Agrawal and Dr. Dheeraj Yadav}

DOI: https://doi.org/10.22271/ortho.2021.v7.i2g.2667

\section{Abstract}

Background: The frozen shoulder is very common problem in India and usually could occur as idiopathic or trivial injury. Management modality is usually conservative and physiotherapy because of self-limiting nature of disease. Role of corticosteroid injection are usually effective for short period time but newer Orthobiologic agent like PRP injection are equally effective and minimal and no side effect.

Material and method: In our study, total 50 patients were taken of adhesive capsulitis in period October 2018 to February 2020 and divided into groups randomly and PRP injection and corticosteroid injection was given and clinical outcome was recorded.

Result: In our study, female are more affected then male, usually occur in age 40 to 60 -year age group. The final outcome at 3 month post injection $20 \%$ patients with excellent, $48 \%$ with good and $32 \%$ with poor outcome with PRP injection group and for CS injection group this was found as $16 \%$ excellent, $36 \%$ good and $48 \%$ poor outcome.

Conclusion: We concluded PRP and MPS showed efficacy and strong evidence in support significant effect of PRP, where steroid contraindicated or refused by patient in the treatment of frozen shoulder. However, inj. Methylprednisolone has sudden onset of action because of anti-inflammatory action with respect to inj. PRP, so has better result at 1 week follow up post injection but in long term (at 3 months follow up) inj. PRP has better effect in compared to injection MPS.

Keywords: PRP, adhesive capsulitis, methyl prednisolone, frozen shoulder

\section{Introduction}

Frozen shoulder is characterized by pain and limitation of movement apparently of spontaneous onset or following slight injury but still its cause is poorly understood and its management is disputed because of lack of supporting evidence ${ }^{[1]}$. The symptoms are generally self-limiting over one to three years and condition more common in females than males and the greatest incidence occurs in the $5^{\text {th }}$ and $6^{\text {th }}$ decades. The X-ray appearances may show either nothing abnormal or calcific deposits in the capsule or periarticular tissue. The Management is mainly focused on restoring joint movement and reducing shoulder pain, using anti-inflammatory medications, physical therapy, injection of saline with LA and/or surgical intervention including myofascial release. Although all these treatment may "unfreeze" the shoulder but usually, do not completely alleviate the chronic pain ${ }^{[2]}$. Another practice of modern medicine is to inject methylprednisolone to prescribe anti-inflammatory medications. Platelet-rich plasma (PRP) is an orthobiologic that has recently gained popularity as an adjuvant treatment for musculoskeletal injuries ${ }^{[3]}$. The platelets contain alpha granules that are rich in several growth factors, such as platelet-derived growth factor, transforming growth factor- $\beta$, insulin-like growth factor, vascular endothelial growth factor and epidermal growth factor, which play key roles in tissue repair mechanisms ${ }^{[4,5]}$. The PRP injection therapy can have a beneficial effect in the management of frozen shoulder. Since there are very few studies evaluating the beneficial effect of PRP injection therapy versus injection methylprednisolone in the treatment of periarthritis shoulder, we conducted a novel study in our institute to assess its results and biological effects. 


\section{Material and Methods}

This is prospective study, we have taken total of 50 patients of frozen shoulder after proper clinical and radiological (X-ray, CT, MRI) assessment period of October 2018 to February 2020 in our institute with their informed consent and explanation regarding procedure and divided them randomly equally into two subgroups: subgroup A received PRP injection while subgroup B had received methylprednisolone injection. The inclusion criteria of this study was adult more than 18 year and stage 2 or more of periarthritis shoulder, shoulder range of motion decrease to $50 \%$ or more than opposite shoulder. The patients who had bilateral periarthritis shoulder, stage 1 or lesser grade, less than 18-year age, any superficial or deep infection, any associated fracture, any comorbid condition, diabetic patients and those who were not willing for injection excluded from our study. The PRP injection was making with withdrawing $20-30 \mathrm{ml}$ of patient venous blood and then with addition of sodium citrate double centrifugation was done at $1500 \mathrm{rpm}$ for 6 minute and then at $3400 \mathrm{rpm}$ for $15 \mathrm{~min}$ for getting high concentration platelet rich plasma. PRP preparation was activate with calcium gluconate, filled into syringe, and inject into affected shoulder of patients of subgroup A (figure 1). The injection of methylprednisolone $2 \mathrm{cc}$ was inserted to affected shoulder in patients of subgroup B. All patients of our study advised neither to take any kind of analgesics i.e. NSAIDS nor any massage of shoulder, if patient had severe pain following injection opioids analgesic like the one tramadol can be given to patients. Patients were followed up at 1 week post injection, then after 1 month and then at 3 months.

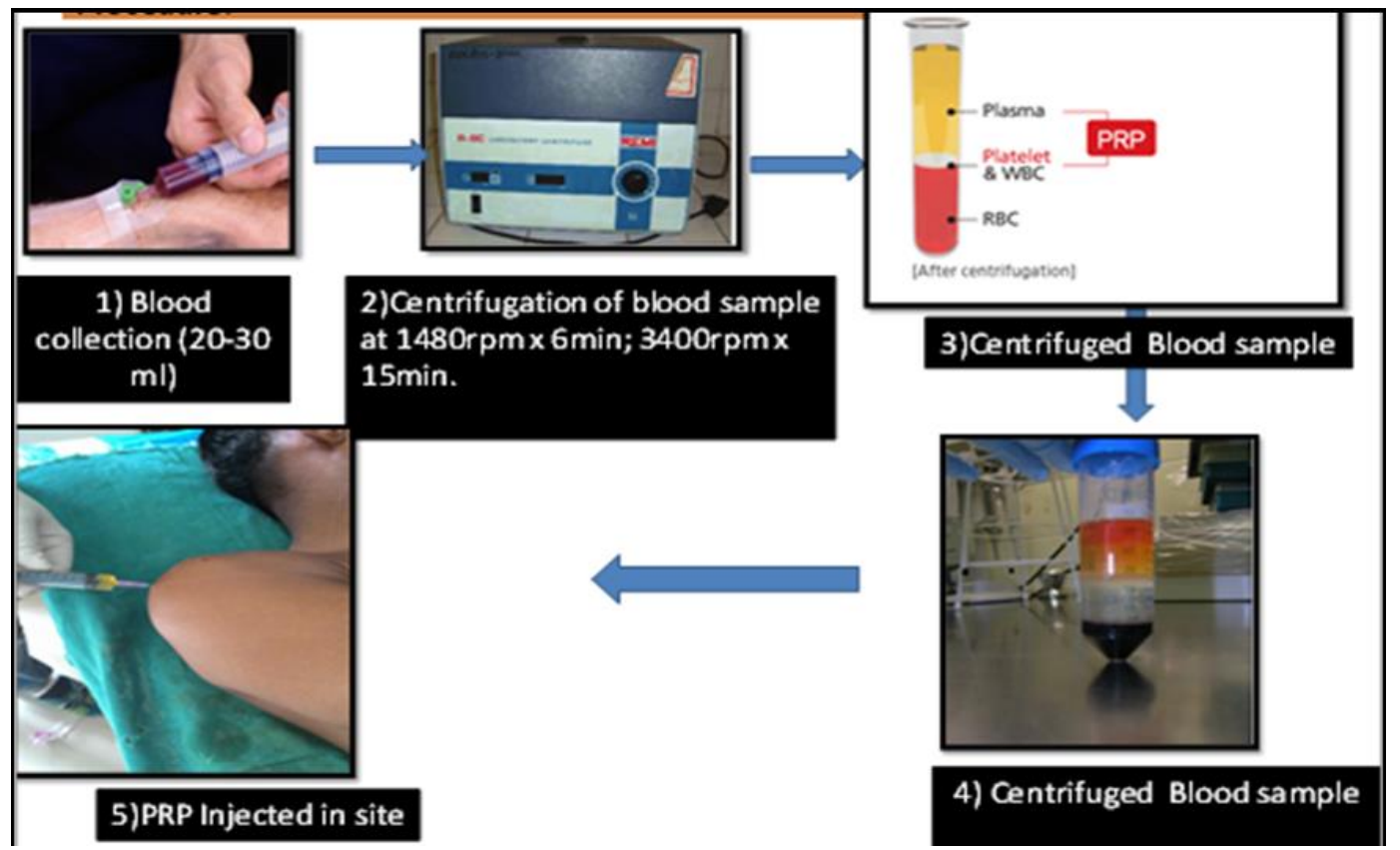

Fig 1: Showing PRP preparation and injection to patients

\section{Result}

In this study, there were $60 \%$ female patients and $40 \%$ were males. Most of the patients are in age group of 41-60 years in both the groups. Most of the patients $(60 \%)$ were injected with PRP in period of 3-6 months and with inj. MPS also in 3-6 months $(56 \%)$. There is statistically significant reduction in VAS pain scores after getting either with PRP injection as well as with MPS injection over 3-6 month period as depicted in Table 1. The comparative clinical outcome during the follow-up period with PRP and MPS injection were given in Table 2 and table 3 respectively and final 3-month follow up suggest $20 \%$ patients with excellent, $48 \%$ with good and $32 \%$ with poor outcome with PRP injection. For subgroup B this was found as $16 \%$ excellent, $36 \%$ good and $48 \%$ poor outcome (table 4). The complication rate as PRP is made of patients own blood, there is no such complications except local site post injection pain seen in 14 patients $(56 \%)$ for some time.

Table 1: Comparisons of pain according to VAS during pre and post treatment

\begin{tabular}{|c|c|c|}
\hline Time interval & PRP $($ Mean \pm SD) & MPS (Mean \pm SD) \\
\hline Pre & $8.76 \pm 0.99$ & $8.48 \pm 0.80$ \\
\hline Post -1 week & $5.60 \pm 2.75$ & $5.92 \pm 2.26$ \\
\hline Post -1 month & $4.00 \pm 3.49$ & $3.92 \pm 2.67$ \\
\hline Post -3 month & $3.12 \pm 3.85$ & $3.12 \pm 3.31$ \\
\hline P value & $<0.00001(\mathrm{~S})$ & $<0.00001(\mathrm{~S})$ \\
\hline Chi-square & 48.216 & 40.968 \\
\hline
\end{tabular}

Table 2: Comparisons of Constant score during pre and post treatment (PRP)

\begin{tabular}{|c|c|c|c|c|c|c|c|c|c|c|c|}
\hline \multirow{2}{*}{ Pre } & \multicolumn{3}{|c|}{ Post - 1 week } & \multicolumn{3}{c|}{ Post - 1 month } & \multicolumn{3}{c|}{ Post - 3 month } & \multirow{2}{*}{ Total } \\
\cline { 2 - 12 } & Good & Fair & Poor & Excellent & Good & Fair & Poor & Excellent & Good & Poor & \\
\hline Fair & 1 & 2 & 0 & 0 & 2 & 1 & 0 & 0 & 3 & 0 & 9 \\
\hline Poor & 4 & 10 & 8 & 1 & 9 & 4 & 8 & 5 & 9 & 8 & 66 \\
\hline Total & 5 & 12 & 8 & 1 & 11 & 5 & 8 & 5 & 12 & 8 & 75 \\
\hline p value & \multicolumn{3}{|c|}{0.440} & \multicolumn{3}{|c|}{0.587} & & & 0.158 & & \\
\hline
\end{tabular}


Table 3: Comparisons of Constant score during pre and post treatment (MPS)

\begin{tabular}{|c|c|c|c|c|c|c|c|c|c|c|c|}
\hline \multirow{2}{*}{ Pre } & \multicolumn{3}{|c|}{ Post - 1 week } & \multicolumn{4}{|c|}{ Post - 1 month } & \multicolumn{3}{|c|}{ Post - 3 month } & \multirow{2}{*}{ Total } \\
\hline & Good & Fair & Poor & Excellent & Good & Fair & Poor & Excellent & Good & Poor & \\
\hline Fair & 3 & 0 & 0 & 1 & 2 & 0 & 0 & 1 & 2 & 0 & 9 \\
\hline Poor & 3 & 6 & 13 & 0 & 8 & 2 & 12 & 3 & 7 & 12 & 66 \\
\hline Total & 6 & 6 & 13 & 1 & 10 & 2 & 12 & 4 & 9 & 12 & 75 \\
\hline $\mathrm{p}$ value & & 0.005 & & & 0.020 & & & & 205 & & \\
\hline
\end{tabular}

Table 4: Comparative final outcome at 3 month post injection follow up

\begin{tabular}{|c|c|c|}
\hline Results & PRP & MPS \\
\hline Excellent & $5(20 \%)$ & $4(16 \%)$ \\
\hline Good & $12(48 \%)$ & $9(36 \%)$ \\
\hline Poor & $8(32 \%)$ & $12(48 \%)$ \\
\hline
\end{tabular}

\section{Discussion}

The primary goal of this study was to evaluate the efficacy of intra-articular PRP and corticosteroid injection in patients with idiopathic adhesive capsulitis. The pathology involved in adhesive capsulitis is synovial hyperplasia and capsular fibroplasia with fibrosis and dense capsular scar formation.

Rodeo et al. reported role of cytokines and other inflammatory mediators in patients with adhesive capsulitis and Intra-articular corticosteroid decreases synovitis limits development of fibrosis ${ }^{[6,7]}$.

Van der Windt et al. compared intra-articular corticosteroid to 6 weeks of physical therapy for patients with painful stiff shoulders and reported significant improvements in pain, disability, and motion in the injection group ${ }^{[8]}$.

Gam et al ${ }^{[9]}$ treated patients with adhesive capsulitis with either steroid injection or saline injection and distension with $19 \mathrm{~cm}^{3}$ of Lidocaine and found that the distension with steroid group (12 patients) used fewer analgesics and had improved motion compared to the steroid-only group (eight patients). There are many disadvantages of corticosteroid injection have been reported including periarticular calcification, cutaneous atrophy, cutaneous depigmentation, tendon rupture, avascular necrosis, and joint infection ${ }^{[10]}$ but in our study, no significant adverse effect have been reported. Thus, corticosteroid injection in the early stages of adhesive capsulitis leads to significant improvement in range of motion and pain. Our study demonstrated that PRP is not inferior to corticosteroid injection in any of the measured parameters and both of the groups experienced similar benefits from the injection therapies with no statistical differences detected in ROM or VAS scores at 1 week, 1 month and 3 months and no adverse effects were detected in either of any two groups. Our results are consistent with current literature, showing that PRP can be beneficial treatment of adhesive capsulitis ${ }^{[11,12]}$. The previous studies are controversial in interpreting the efficacy of PRP injections due to the different research and treatment protocols, in many cases involving arthroscopy or different products of PRP, for example PRP fibrin matrix ${ }^{[13,14]}$ and retrospective design and lack of randomization might have been the major limitations of this study $[15,16]$. The current literatures strongly advices against surgery in conditions like frozen shoulder and favors conservative treatment options ${ }^{[17]}$. In this perspective, PRP may offer a valid alternative to corticosteroid injection, considering that there are no documented significant adverse effects in PRP treatments unlike in corticosteroid injection treatments [11]. The advantages of PRP over CS are the absence of severe complications locally and systematically and more safe and simple treatment while disadvantages of PRP would be more injections required achieving similar outcomes as a single corticosteroid injection. The PRP treatment may be repeated whether symptoms return, but multiple corticosteroid injection should be avoided and concurrent physical therapy is still advised because of its proven benefits, as seen with given the outcomes of our study, we recommend considering PRP as an alternative treatment to CS in order to reduce local and systemic effects involved with CS injections.

\section{Conclusion}

We can conclude that both PRP and MPS showed good efficacy on treating frozen shoulder. The current study provides strong evidence in support of a statistically significant effect of platelet concentrate in the treatment of frozen shoulder in vivo where steroid contraindicated or refused by patient. However, inj. Methylprednisolone has sudden onset of action because of anti-inflammatory action with respect to inj. PRP, so has better result at 1 week follow up post injection but in long term (at 3 months follow up) inj. PRP has better effect in compared to injection MPS.

Source of support: Nil

Conflict of interest: Nil

Informed consent: taken from each patient

\section{References}

1. Chambler AFW, Carr AJ. Aspects of current management. The role of surgery in frozen shoulder. J Bone Joint Surg [Br] 2003;85-B:789-95.

2. Andews JR. Diagnosis and treatment of chronic painful shoulder: Review of nonsurgical interventions. Journal of Arthroscopic and Related Surg 2005;21(3):333-347

3. Dhillon RS, Schwarz EM, Maloney MD. Platelet-rich plasma therapy - future or trend? Arthritis Res Ther 2012;14(4):219.

4. Alsousou J, Ali A, Willett K, Harrison P. The role of platelet-rich plasma in tissue regeneration. Platelets, 2012.

5. Bava ED, Barber FA. Platelet-rich plasma products in sports medicine. Phys Sportsmed. 2011;39(3):94-9.

6. Rodeo SA, Hannafin JA, Tom J, Warren RF, Wickiewicz TL. Immunolocalization of cytokines and their receptors in adhesive capsulitis of the shoulder. J Orthop Res 1997; 15:427-436.

7. Bunker TD, Anthony PP. The pathology of frozen shoulder. A Dupuytren-like disease. J Bone Joint Surg Br 1995;77:677-683.

8. Vander Windt DAWM, Koes BW, Deville W, Boeke AJP, de Jong BA, Bouter LM. Effectiveness of corticosteroid injections versus physiotherapy for treatment of painful stiff shoulder in primary care. BMJ 1998;317:1292-1296.

9. Gam AN, Schydlowsky P, Rossel I, Remvig L, Jensen EM. Treatment of "frozen shoulder" with distension and glucorticoid compared with glucorticoid alone, a randomized controlled trial. SC and $\mathrm{J}$ Rheumatol 1998;27:425-430.

10. Habib GS. Systemic effects of intra-articular 
corticosteroids. Clin Rheumatol 2009;28:749-756.

11. Shams A, El-Sayed M, Gamal O, Ewes W. Subacromial injection of autologous platelet-rich plasma versus corticosteroid for the treatment of symptomatic partial rotator cuff tears. Eur. J Orthop Surg Traumatol 2016;26:837-842.

12. Von Wehren L. The effect of subacromial injections of autologous conditioned plasma versus cortisone for the treatment of symptomatic partial rotator cuff tears. Knee Surg Sports Traumatol Arthrosc 2016;24:3787-3792.

13. Hurley ET, Lim Fat D, Moran CJ, Mullett H. The efficacy of platelet-rich plasma and Plateletrich Brin in arthroscopic rotator cuff repair: A meta-analysis of randomized controlled trials. Am J Sports Med 2019;47:753-761.

14. Randelli P, Arrigoni P, Ragone V, Aliprandi A, Cabitza P. Platelet rich plasma in arthroscopic rotator cuff repair: a prospective RCT study, 2-year follow-up. J Shoulder Elbow Surg 2011;20:518-528.

15. Lewis J. Rotator cuff related shoulder pain: Assessment, management and uncertainties. Man Ther 2016;23:57-68.

16. Diercks $\mathrm{R}$ et al. Guideline for diagnosis and treatment of subacromial pain syndrome: a multidisciplinary review by the Dutch Orthopaedic Association. Acta Orthop 2014;85:314-322.

17. Ketola S, Lehtinen JT, Arnala I. Arthroscopic decompression not recommended in the treatment of rotator cuff tendinopathy: a final review of a randomised controlled trial at a minimum follow-up of ten years. Bone Joint J 2017;99-B:799-805. 